\title{
Measurements of the Higgs boson decays to two bottom quarks
}

\author{
Luca Ambroz*, \\ on behalf of the ATLAS Collaboration \\ Department of Physics \\ University of Oxford \\ E-mail: luca.ambroz@cern.ch
}

$H \rightarrow b \bar{b}$ decays make it possible to probe the Yukawa coupling of the Higgs boson to down type quarks. Observing these processes at the LHC is extremely challenging due to the large multi-jet background; however, this can be greatly suppressed by triggering on missing transverse energy or charged leptons coming from the decay of a weak vector boson produced together with the Higgs boson. In this paper, the latest measurement for $H \rightarrow b \bar{b}$ decays associated with a $W$ or $Z$ boson with the ATLAS detector will be presented. Furthermore, the first $V H, H \rightarrow b \bar{b}$ differential measurement in bins of transverse momentum of the vector bosons will be discussed; this type of differential measurement is particularly sensitive to Beyond Standard Model physics, as probed through an Effective Field Theory approach.

XXIX International Symposium on Lepton Photon Interactions at High Energies - LeptonPhoton2019 August 5-10, 2019

Toronto, Canada

${ }^{*}$ Speaker. 


\section{Introduction}

In 2012 the ATLAS and CMS experiments [1,2] at the LHC claimed the observation of a new particle compatible with the production and decay of the Standard Model Higgs boson [3, 4]. The decay channels primarily used for this discovery in ATLAS were $H \rightarrow \gamma \gamma, H \rightarrow Z Z^{*}$ and $H \rightarrow W W^{*}$. A fit to the signals extracted from these channels allowed a first mass measurement of approximately $125 \mathrm{GeV}$.

The measurement of the Higgs boson mass fixed all the Standard Model branching ratios. In particular, for $m_{H} \simeq 125 \mathrm{GeV}$, the decay of the Higgs boson into two bottom quarks has the largest branching ratio. The observation of this Higgs boson final state is hampered by many experimental challenges, among which the biggest is the small signal-to-background ratio. To suppress the overwhelming number of background events, the Higgs boson is sought in association with an electroweak vector boson decaying into two leptons. The production cross-section of associated Higgs boson production is more than an order of magnitude smaller than the $p p \rightarrow H$ production via gluon-gluon fusion, as can be seen in Figure 1; however, with the data collected between 2015 and 2017 at $\sqrt{s}=13 \mathrm{TeV}$ the ATLAS collaboration manage to measure this elusive decay.

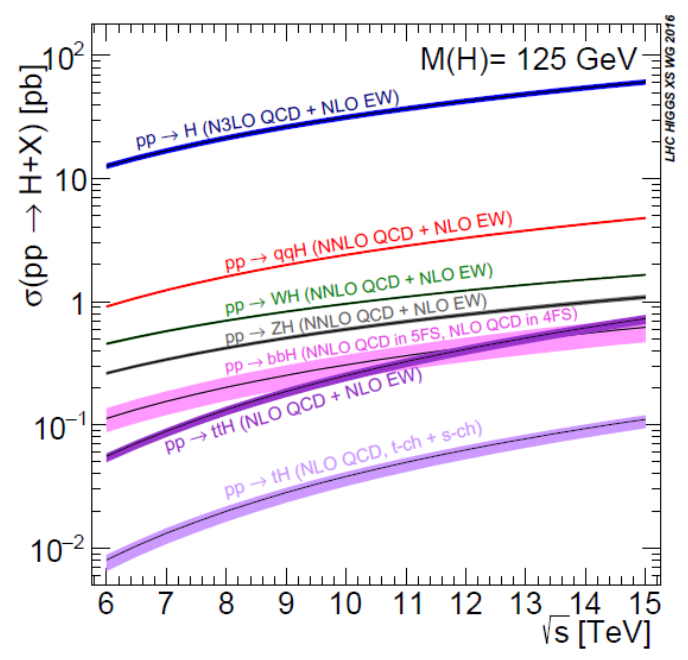

(a)

Figure 1: Higgs boson production cross-sections at LHC as a function of the centre-of-mass energy [5].

\section{Observation of $\mathbf{H} \rightarrow \mathbf{b} \overline{\mathbf{b}}$ decays}

The $V H$ production is the most sensitive production mode for the discovery of the Higgs boson decaying in two bottom quarks. The ATLAS $V H, H \rightarrow b \bar{b}$ analysis with the 2015-2017 data will be described in this section [6].

Events are categorised in lepton channels according to the number of charged leptons (electrons and muons) that are present. Events are further divided in 2-jet or 3-jet categories according to the total number of jets. In the 2-lepton channel, the 3-jet category includes events with three or more jets. Signal regions are defined for the reconstructed transverse momentum $p_{\mathrm{T}}^{V}$ (or transverse 
missing momentum $E_{\mathrm{T}}^{\mathrm{miss}}$ in the 0-lepton channel) greater than $150 \mathrm{GeV}$. In the 2-lepton channel one additional region has been added for $75 \mathrm{GeV}<p_{\mathrm{T}}^{V}<150 \mathrm{GeV}$. All events are required to have exactly two jets passing the b-tagging requirement, and one additional non-b-tagged jet is allowed (even more in the 2-lepton channel). Jets are reconstructed with the anti- $k_{t}$ algorithm with radius 0.4 [7]. Across all categories, at least one of the b-jets must have transverse momentum $p_{\mathrm{T}}>45$ $\mathrm{GeV}$.

$E_{\mathrm{T}}^{\text {miss }}$ triggers have been used with thresholds that vary according to the LHC operating instantaneous luminosity in the 0-lepton channel and in the 1-lepton muon sub-channel. Conversely, single-lepton triggers have been used in the 1-lepton electron sub-channel and 2-lepton channel.

For the 0-lepton channel, a selection on the scalar sum of the transverse momenta of all the jets in the event, $H_{\mathrm{T}}$, is placed. The requirement is $H_{\mathrm{T}}>120 \mathrm{GeV}\left(H_{\mathrm{T}}>150 \mathrm{GeV}\right)$ for 2-jet (3-jet) events. This cut is necessary to remove a portion of the phase space in which the trigger efficiency depends on the jet multiplicity. Lastly, requirements on the angular distance between reconstructed objects are used to suppress the QCD multijet background in the 0-lepton channel. These requirements exploit the fact that in signal events the vector boson tends to be produced back-to-back with the Higgs boson.

In the 1-lepton channel, a single lepton is required satisfying isolation criteria and minimum $p_{\mathrm{T}}$ requirements. Control regions (Figure 2(a)) are created to evaluate our understanding of the background due to $W$ bosons produced in association with jest containing heavy-flavour hadrons $(W+\mathrm{HF})$ by introducing additional cuts on the reconstructed invariant mass of the $\mathrm{b} \bar{b}$-system $\left(m_{b \bar{b}}<75 \mathrm{GeV}\right)$ and on the reconstructed top mass $\left(m_{\mathrm{top}}>225 \mathrm{GeV}\right)$.

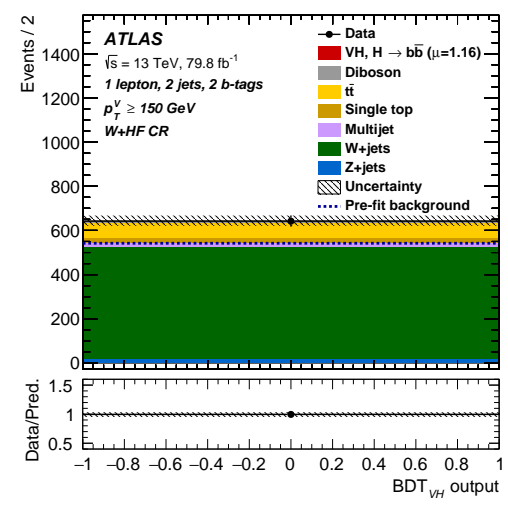

(a)

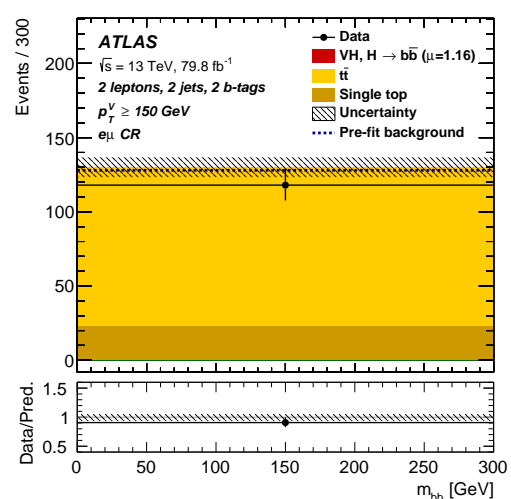

(b)

Figure 2: Control regions for the $W+$ HF background in the 1-lepton (a) and $t \bar{t}$ background in the 2-lepton (b) channels for 2-jet events with with $p_{\mathrm{T}}^{V}>150 \mathrm{GeV}$ [6].

Lastly, for the 2-lepton channels, the selection is similar to the 1-lepton channel with the addition of one more same flavour lepton and an additional cut on the invariant mass of the two leptons within $10 \mathrm{GeV}$ of the $Z$-mass. The 2-lepton channel has also a very pure control regions to constrain the $t \bar{t}$ background by requiring events with one electron and one muon (Figure 2(b)).

Once the events have been selected and categorised, Boosted Decision Trees (BDTs) are trained on simulated events for every signal region in the analysis to discriminate signal from background events. The BDTs output score $\left(\mathrm{BDT}_{\mathrm{VH}}\right)$ for the expected signal and background events 
from simulation as well as data events is evaluated and fitted to extract the signal strength $\mu$ of the $V H, H \rightarrow b \bar{b}$ production (Figure 3 ). In the $W+$ HF control regions only the event yields are fitted, whereas in the top control regions the event yield is fitted for the 2-jet region with $p_{\mathrm{T}}^{V}>150 \mathrm{GeV}$ (due to limited data statistics), while $m_{b \bar{b}}$ is fitted for all the other ones (Figure 2).

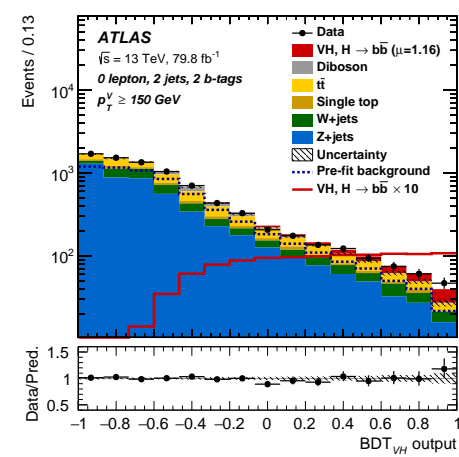

(a)

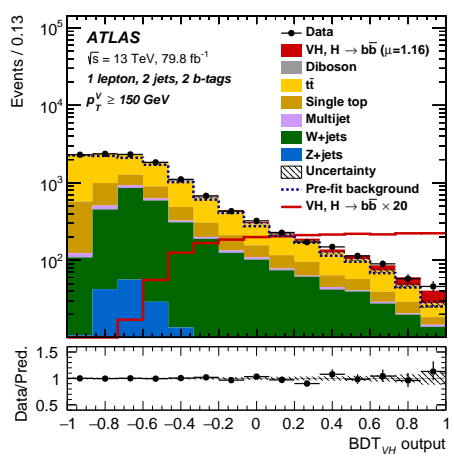

(b)

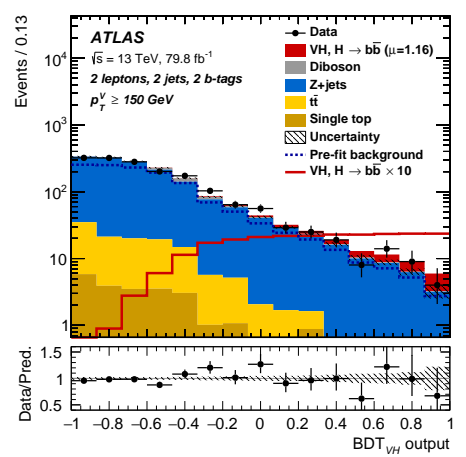

(c)

Figure 3: Post-fit $\mathrm{BDT}_{\mathrm{VH}}$ output distributions for the signal regions in 0-lepton (a), 1-lepton (b) and 2-lepton (c) channels for 2-jet events with with $p_{\mathrm{T}}^{V}>150 \mathrm{GeV}[6]$.

The result of the fit with $79.8 \mathrm{fb}^{-1}$ of data is $\mu_{V H, H \rightarrow b \bar{b}}=1.16_{-0.25}^{+0.27}$ with an observed significance of 4.9 standard deviations and an expected significance of 4.3 standard deviations. This result is compatible with the Standard Model within the uncertainties. When this $V H \rightarrow b \bar{b}$ Run 2 measurement is combined with the Run 1 measurement and with all other Higgs production modes probed in $H \rightarrow b \bar{b}$ searches, the result is the observation of the Higgs boson decays into a pair of bottom quarks, with 5.4 standard deviations (Figure 4(a)).

Similarly, the ATLAS Run 2 measurements involving the $V H$ production are combined, resulting in the observation of the $V H$ production mode, with 5.3 standard deviations (Figure 4(b)).

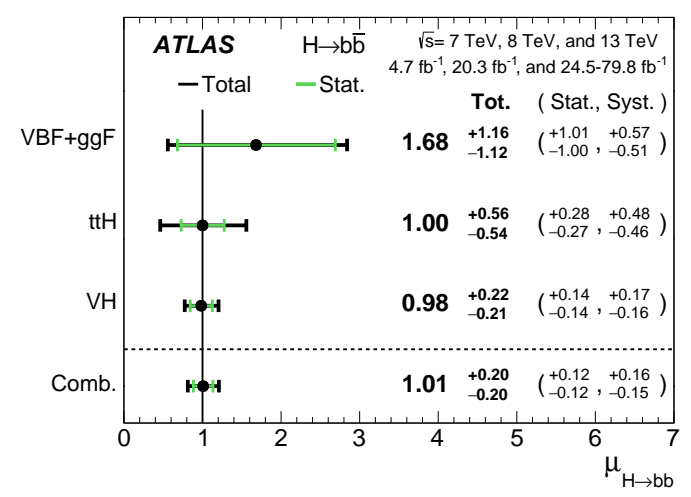

(a)

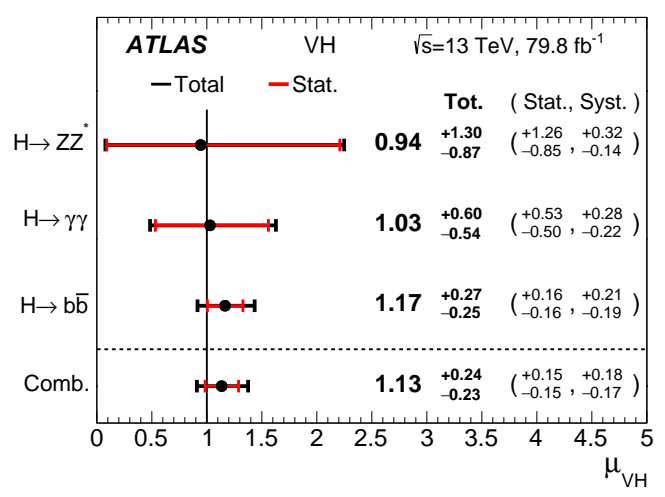

(b)

Figure 4: Measured post-fit signal strengths for a Higgs boson of mass $m_{H}=125 \mathrm{GeV}$ decaying in two bottom quarks (a) and produced in association with a vector boson (b) [6]. 


\section{First differential $\mathrm{VH}, \mathrm{H} \rightarrow \mathbf{b} \overline{\mathbf{b}}$ measurement}

The $V H, H \rightarrow b \bar{b}$ analysis probes $V H$ production with the greatest sensitivity compared to the other decay channels, as can be seen from Figure 4(b). This is possible because the large branching ratio of $H \rightarrow b \bar{b}$ decay compensates the small $V H$ production cross-section. Thus, the $V H, H \rightarrow b \bar{b}$ analysis, which was described in Section 2, has been expanded in a cross section measurement as a function of the weak boson transverse momentum in kinematic fiducial volumes according to the Simplified Template Cross Section (STXS) framework [8]. This differential analysis keeps the event selection and BDT trainings of the inclusive analysis described in the previous section. The cross sections are measured in five regions according to the Truth STXS $p_{\mathrm{T}}^{V}$ [9], two for WH production and three for $\mathrm{ZH}$ production:

- $W H$ production: $150 \mathrm{GeV}<p_{\mathrm{T}}^{W}<250 \mathrm{GeV}$ and $250 \mathrm{GeV}<p_{\mathrm{T}}^{W}$;

- $\mathrm{ZH}$ production: $75 \mathrm{GeV}<p_{\mathrm{T}}^{\mathrm{Z}}<150 \mathrm{GeV}, 150 \mathrm{GeV}<p_{\mathrm{T}}^{\mathrm{Z}}<250 \mathrm{GeV}$ and $250 \mathrm{GeV}<p_{\mathrm{T}}^{Z}$;

The BDT $\mathrm{VH}$ output distributions of the truth STXS templates in these regions differ from each other for a given region in reconstructed $p_{\mathrm{T}}^{V}$, as can be seen in Figure 5; these differences provide discriminating power to the fit. The five measured cross sections times branching ratio are reported in Figure 6(a). These measurements are consistent with the Standard Model predictions.

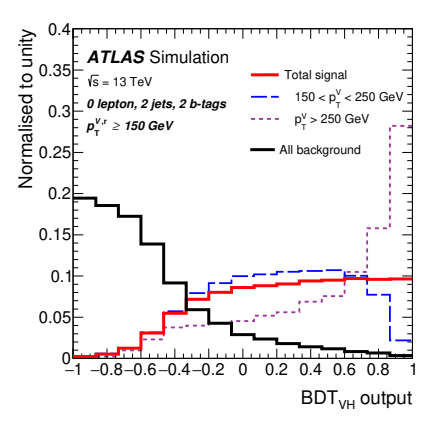

(a)

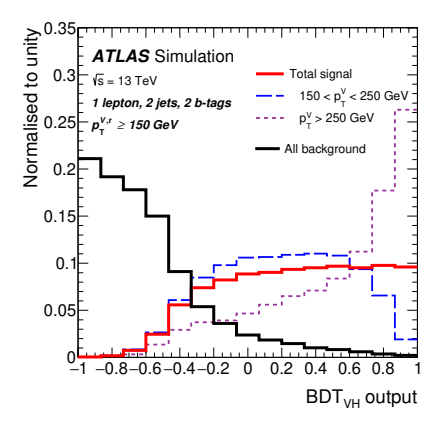

(b)

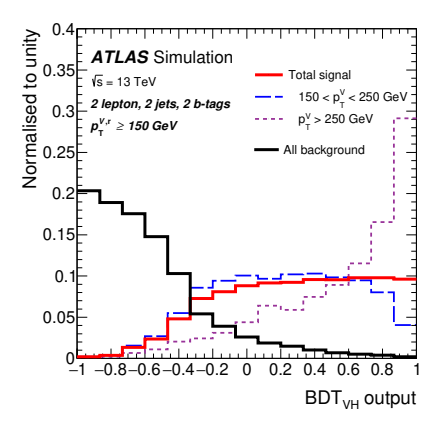

(c)

Figure 5: $\mathrm{BDT}_{\mathrm{VH}}$ output shapes for different $p_{\mathrm{T}}^{V}$ Truth STXS templates for the 0-lepton (a), 1-lepton (b) and 2-lepton (c) channels for 2-jets events with with reconstructed $p_{\mathrm{T}}^{V}>150 \mathrm{GeV}$ [8].

The Standard Model Lagrangian can be expanded with an Effective Field Theory parametrisation to describe Beyond Standard Model physics by adding higher-order terms [10]. Assuming no contributions from dimension-5 operators, which would violate the lepton or baryon number, and from dimension-7 (or greater) operators, which would be further suppressed from the energy scale of the interaction, the Lagrangian can be written as:

$$
\mathcal{L}=\mathcal{L}_{S M}+\mathcal{L}_{B S M} \simeq \mathcal{L}_{S M}+\frac{1}{\Lambda^{2}} \sum_{i} \bar{c}_{i} \mathcal{O}_{i}+\ldots
$$

where $\Lambda$ represents the energy scale of the interaction and $\bar{c}_{i}$ are the dimensionless coupling constants (Wilson coefficients) to new operators $\mathcal{O}_{i}$ calculated with the SILH basis [10]. 
The cross-sections measured in the fiducial bins are particularly sensitive to some Wilson coefficients, as can be seen on Figure 6(b). Thus, limits at 95\% confidence level on these coefficients can be placed by performing one-dimensional fits in which only one of the coefficients is kept floating and all the others are set to zero (Table 1).

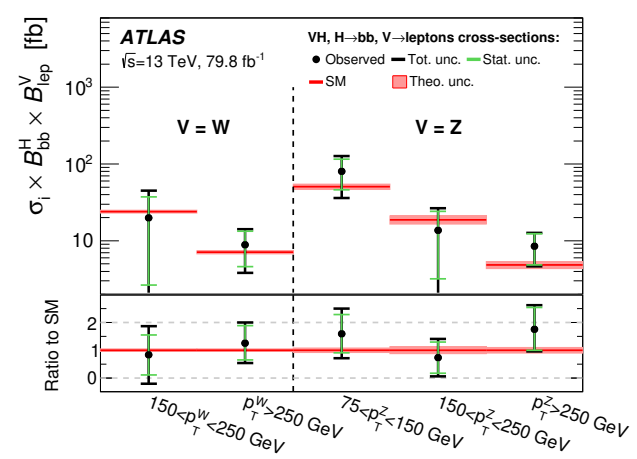

(a)

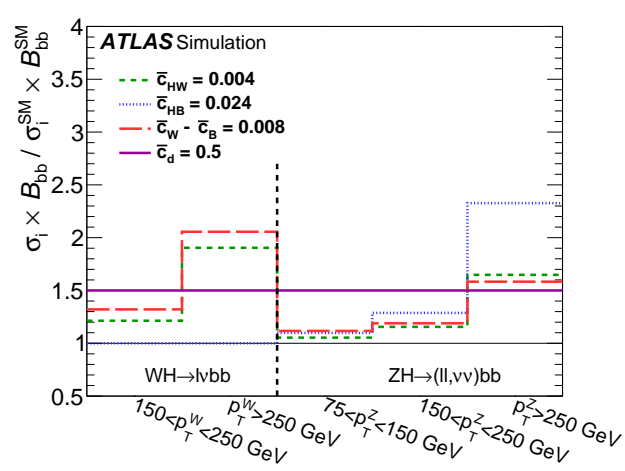

(b)

Figure 6: Measured cross sections times $H \rightarrow b \bar{b}$ and $V \rightarrow$ leptons branching ratio for the five fiducial regions (a) and impact on the cross sections for Wilson coefficients (Table 1) that are expected to be excluded at $95 \%$ confidence level (b) [8].

\begin{tabular}{ccc}
\hline \hline Coefficient & Expected interval & Observed interval \\
\hline \hline $\bar{c}_{H W}$ & {$[-0.018,0.004]$} & {$[-0.019,0.010] \cup[-0.005,0.006]$} \\
\hline $\bar{c}_{H B}$ & {$[-0.078,0.024]$} & {$[-0.090,0.032]$} \\
\hline $\bar{c}_{W}-\bar{c}_{B}$ & {$[-0.034,0.008]$} & {$[-0.036,-0.024] \cup[-0.009,0.010]$} \\
\hline $\bar{c}_{d}$ & {$[-1.7,0.5]$} & {$[-1.9,0.7]$} \\
\hline
\end{tabular}

Table 1: Expected and observed 95\% confidence level intervals for the effective Lagrangian coefficients $\bar{c}_{H W}, \bar{c}_{H B}, \bar{c}_{W}-\bar{c}_{B}$ and $\bar{c}_{d}$ when the other coefficients are fixed to zero [8].

\section{References}

[1] ATLAS Collaboration, JINST 3 (2008) S08003.

[2] CMS Collaboration, JINST 3 (2008) S08004.

[3] ATLAS Collaboration, Phys. Lett. B 716 (2012) 1, arXiv:1207.7214 [hep-ex].

[4] CMS Collaboration, Phys. Lett. B 716 (2012) 30, arXiv:1207.7235 [hep-ex].

[5] LHC Higgs Cross Section Working Group, (2011) Chapters 9 and 13, arXiv:1101.0593 [hep-ph].

[6] ATLAS Collaboration, Phys. Lett. B 786 (2018) 59, arXiv:1808.08238 [hep-ex].

[7] M. Cacciari, G. P. Salam and G. Soyez, JHEP 04 (2008) 063, arXiv: 0802.1189 [hep-ph].

[8] ATLAS Collaboration, JHEP 05 (2019) 141, arXiv:1903.04618 [hep-ex].

[9] LHC Higgs Cross Section Working Group, (2016) Chapter II.1, arXiv:1610.07922 [hep-ph].

[10] R. Contino, M. Ghezzi, C. Grojean, M. Mühlleitner and M. Spira, JHEP 07 (2013) 035, arXiv: 1303.3876 [hep-ph]. 